\title{
Self-Helpingly Exchanging and Charging Batteries for EN-V
}

\author{
Shuping Chen ${ }^{1,}$, Qingchun Zhang ${ }^{1, b}$, Xiangfei Meng ${ }^{1}$, \\ Fenghou Pan ${ }^{1}$, Honglei Liu ${ }^{1}$ and Fanqiang Cheng ${ }^{1}$ \\ ${ }^{1}$ School of Mechanical Engineering and Automation, Northeastern University, Shenyang 110819, \\ China
}

ashpchen@mail.neu.edu.cn (corresponding author), bqczhang71@yahoo.cn

Keywords: EN-V, Battery exchange, Charge, Maltese cross, Self-service, Demo system

\begin{abstract}
An idea of self-helpingly exchanging and charging batteries is proposed to solve some Battery Exchanging and Charging (BEC, for short) problems of Electric-Networked Vehicles (short for, EN-V). Large scale BEC stations occupy large working area and need high equipment cost; common charge piles have disadvantages of long charging time. As a result, a physical demonstration system, which encompasses intelligent vehicle system, BEC stake system and GPS (Global Positioning System) simulation system, is established to validate the feasibility of the idea.
\end{abstract}

\section{Notion of Self-service BEC Stake System}

Electric Vehicles (EV, for short) get their power supply through BEC equipments. The pattern of energy supplement can be divided into vehicle charging (including fast charge, regular charge and slow charge) and battery quick exchange. Generally speaking, large scale BEC stations have characteristics of large occupation of land, high investment and long charging time. Taking Beijing Olympic bus charging station [1] for example, it occupies an area of $5000 \mathrm{~m}^{2}$ and takes up to 2 hours to complete one charge or 10 to 15 minutes to exchange one battery. Another instance is Shenzhen Universidad center charge station [2] which takes up an area of $1092 \mathrm{~m}^{2}$ and consumes an investment of 10.5 million Yuan. 9 hours should be taken to fulfill the charge of hybrid vehicles in the slow charge pattern of the latter station. Commonly, charge piles, with shortcomings of long charging time, occupy smaller areas of land, which makes it flexible to distribute them in large cities. Customers can help themselves to charge by using charge piles, but they may have to wait 6-8 hours to fill up a weak battery. Additionally, a charge pile can charge two or more vehicles simultaneously.

Self-service BEC stake system is a novel idea of EV power supply by way of exchanging batteries. Applying the idea, users can exchange their EV batteries just like shopping in a self-service vending machine, for BEC device of BEC stake system can replace its strong battery with a weak battery in EV, and charge the replaced weak battery. The BEC stake system combines merits of large scale BEC stations and charge piles, and with advantages of small occupation of land, not too much investment and easy construction, it can be constructed comprehensively like building self-service vending machines. On this condition and with the aid of GPS and wireless network, EN-V can real-timely obtain the position information of nearby BEC stake spots and the battery supply information of those spots, and then achieve battery exchange self-helpingly.

As shown in Fig. 1, the BEC stake system commonly encompasses a BEC mechanical infrastructure, a battery exchange control module, a battery information monitor module, an intelligence battery charge module and a network service module. The mechanical infrastructure is used to store spare batteries and exchange them with weak batteries on EV through a mechanical arm 
under the control of the battery exchange control module. The battery information monitor module conducts to supervise charging status (charging or finished) and spare number etc. of batteries, and upload these information to the network service module for users' inquiry. Additionally, an operation display faceplate can be added to the monitor module in order to make users to serve themselves. Just as the name implies, the intelligent battery charge module plays a role in charging weak batteries automatically. When an EV needs new batteries, the first thing for it to know is the position and battery information of BEC stake spots. Therefore, the EV should equip an on-vehicle GPS module and a network service module to acquire those information that can be displayed on an on-vehicle display module for users. In order to arrive a BEC stake spot successfully, an on-vehicle monitor module is required to monitor the quantity of EV battery. A block diagram of EV control system is shown in Fig. 2.

Some mechanical and communicating interfaces are essential to link the BEC stake system and the EV control system. Mechanical interfaces mainly refer to battery compartments of the BEC mechanical infrastructure and EV determined by batteries' fundamental dimensions and hooking part relating to geometric element of hooked part. Communicating interfaces undertake the work of exchanging information between BEC stake system and EV.

For the sake of validating the feasibility of self-service BEC stake system and participating in Freescale Cup University Smart Car Racing Contest in 2011, a physical demonstration system is constructed to experience the idea in the lab.

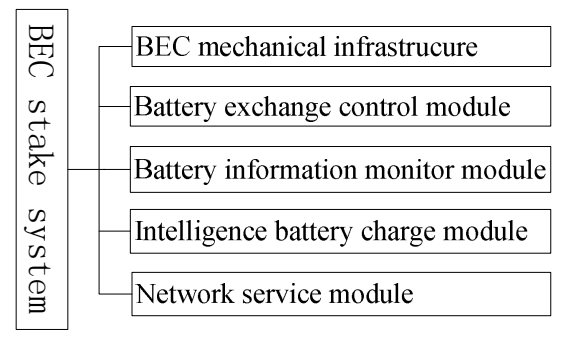

Fig. 1. Block diagram of BEC stake system

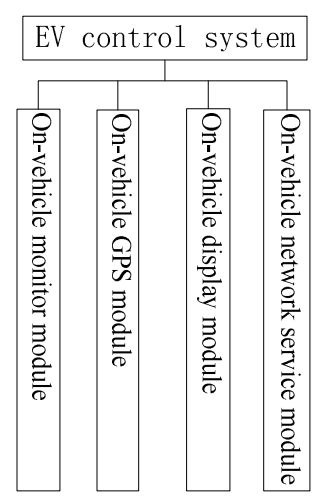

Fig. 2. Block diagram of EV control system

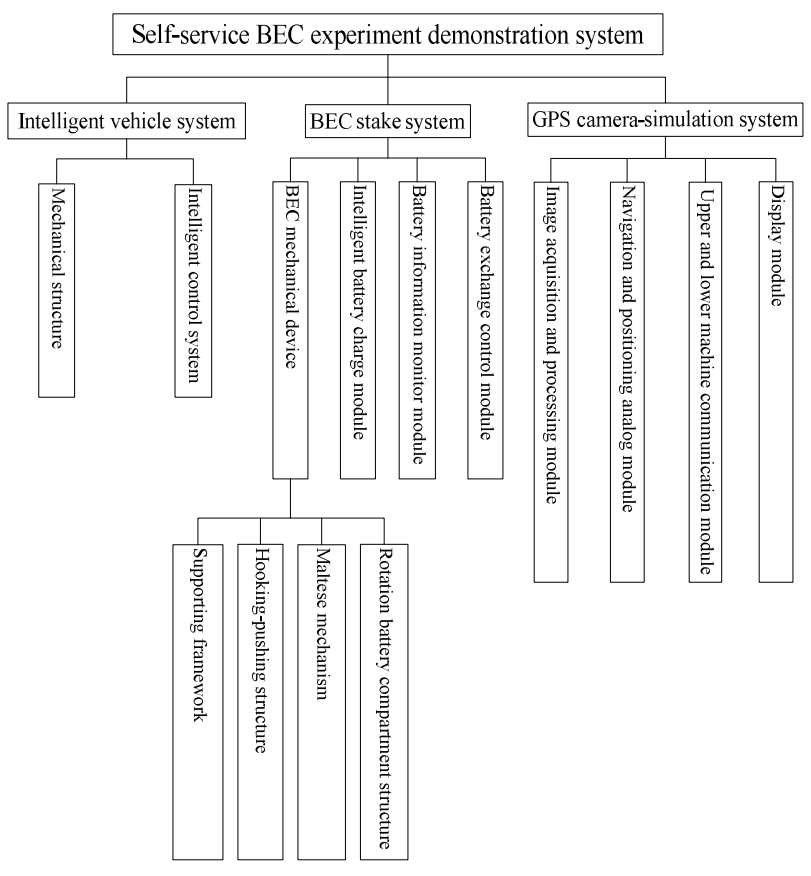

Fig. 3. Scheme of the demo system

\section{Physical Demonstration System}

The demo system chiefly contains intelligent vehicle system, BEC stake system and GPS camera-simulation system, all of which are connected by communications network. Fig. 3 displays the scheme of the self-service BEC experiment demonstration system.

The demo system uses vehicles from the Freescale contest as EV whose control system consists of road detection unit, PID motor control unit, servo control unit, wireless data transmission module and motor lock control unit. As Fig. 3 reveals, the BEC stake system of the demo system is composed 
of BEC mechanical device, intelligent battery charge module, battery information monitor module and battery exchange control module. The GPS simulation system comprises image acquisition and processing module, navigation and positioning analog module, upper and lower machine communication module and display module.

\section{Structure of BEC Device}

The mechanism of BEC device primarily includes Hooking-Pushing (refer with: H-P) structure, rotation battery compartment structure and Maltese cross. The virtual and physical overall structures of BEC device are separately shown in Fig. 4 and Fig. 5.

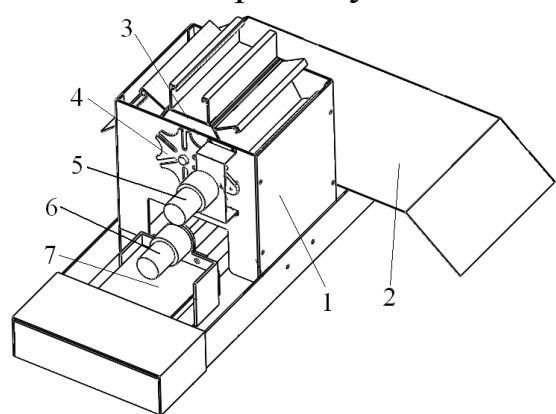

1. support frame, 2. parking platform, 3. rotation battery compartment structure, 4 . Maltese cross, 5. motor III, 6. motor I, 7. H-P structure

Fig. 4. Virtual overall structure of BEC device

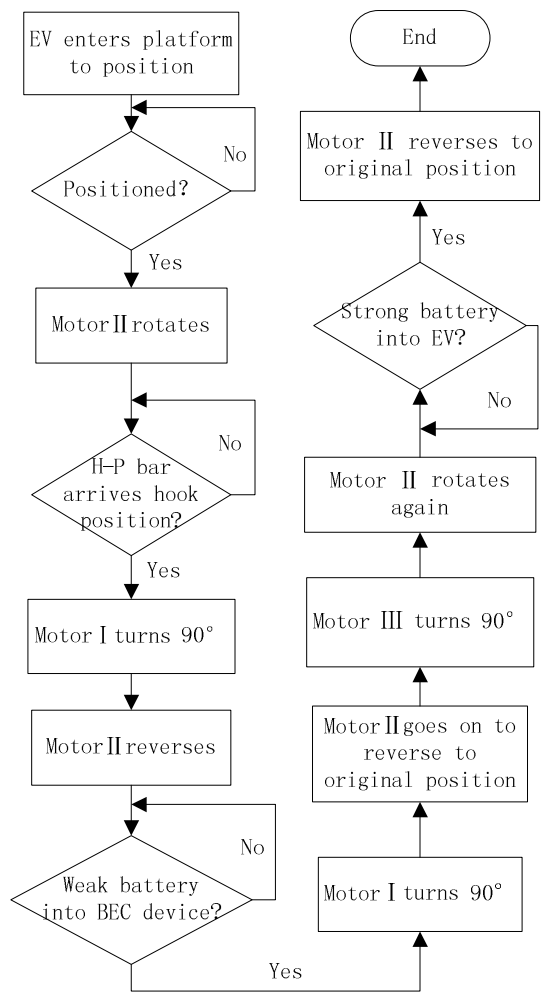

Fig. 6. Work process of BEC device

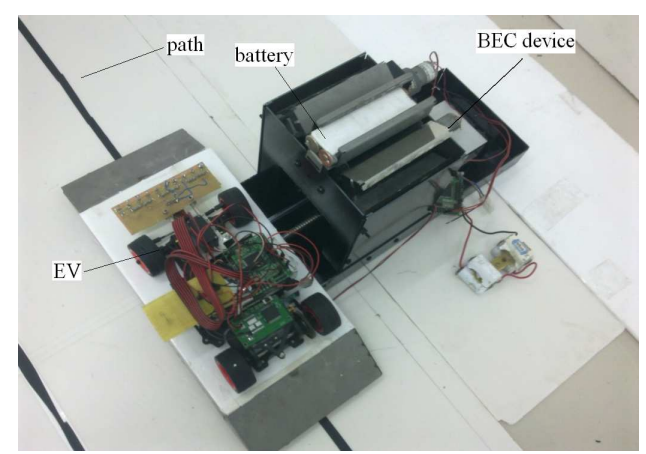

Fig. 5. Physical overall structure of BEC device
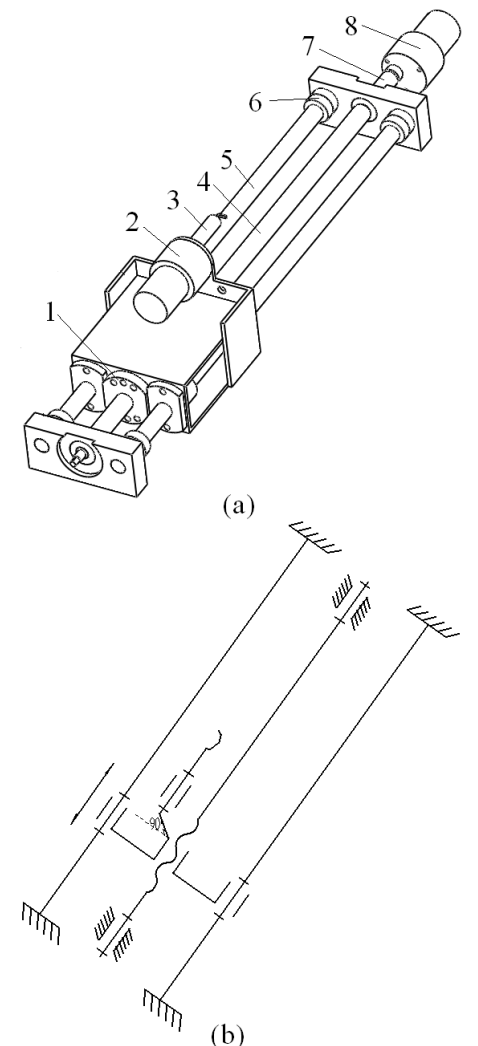

(b)

1. ball-nut, 2. motor I, 3. H-P bar, 4. ball-screw, 5. guide bar, 6. safe pad, 7. shaft coupling, 8 . motor II

Fig. 7. H-P structure 
The work process of BEC device can be seen in Fig. 6 and is explicated as follows: when EV has positioned and parked on parking platform, an empty battery compartment of the rotation battery compartment structure will right face to the on-vehicle battery compartment port to port. Then motor II rotates under the control of the control system to drive ball-screw roll, so that ball-nut and related frameworks can move linearly towards the EV battery. When H-P bar has arrived at the right position, motor I turn 90 degrees in order to make the L-shaped hooker catch on the hook pendant. (Fig. 8) Subsequently, motor II reverses making the H-P bar move back until the weak battery moves into the empty battery compartment. Meanwhile, linear electrodes on the battery contact and conduct the spring lamination of battery compartment and get through the intelligence charge module. Afterwards, motor II stops reversing and motor I makes a $90^{\circ}$ reversal. After that, motor II goes on to invert until the $\mathrm{H}-\mathrm{P}$ bar arrives its original position. Then the grooved pulley turns $60^{\circ}$ correspondingly while motor III rotates to drive the crank move a circle. A battery compartment with a strong battery in it will aim at the on-vehicle battery compartment after the former step. Making motor II rotate again, the H-P bar will push the strong battery into the on-vehicle battery compartment and EV power supply will be conducted as motioned above. A battery exchange process comes to an end when motor II drives H-P frameworks back to the original position again.

The control of a BEC device includes controls of the three motors and the intelligent charge module. A 16-bits Freescale single chip L298 [3] can be used to meet demands of BEC control system. The major action of the EV model is to run automatically on the track and come into a BEC spot to exchange battery under the control of positioning and navigation module when necessary to replace battery.

H-P Structure. Fig. 7(a) exposes components of the H-P structure. An L-shaped hooker can be seen at the end of the H-P bar that connects with motor I. The L-shaped hooker plays an important role in exchanging batteries. Fig. 7(b) shows a mechanism schematic of the H-P structure which makes it convenient to judge the rationality of the structure.

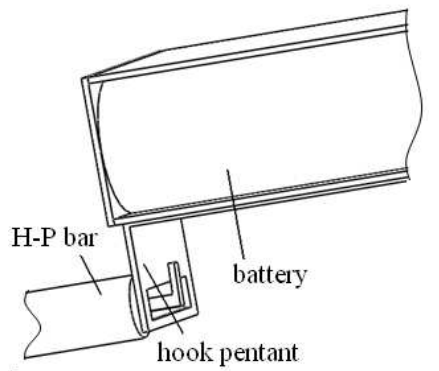

Fig. 8 Hook state

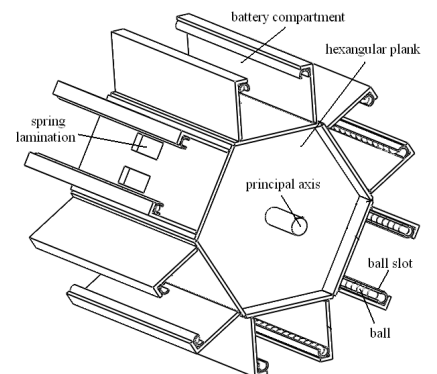

Fig. 9 Rotation battery compartment

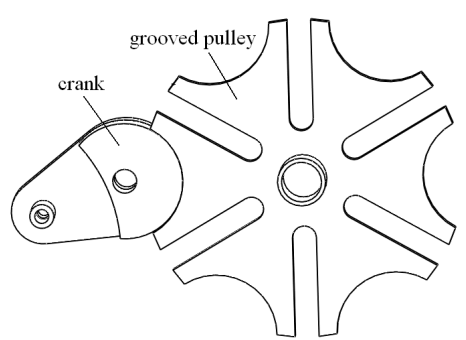

Fig. 10 Maltese cross

Rotation Battery Compartment Structure. As shown in Fig. 9 the rotation battery compartment structure is mainly composed of principal axis, hexangular planks, six same battery compartments, spring laminations, balls and ball slots. Two hexangular planks are parallel and installed at the corresponding position of principal axis. And the six battery compartments are welded symmetrically on the perimeters of the two hexangular planks. At the bottom of each battery compartment, two rows of balls are laid in ball slots in order to reduce the friction of replacing the battery. At the ceiling of the battery compartment, a pair of spring laminations is assembled to ensure the charge circuit turns on automatically when the battery is installed in the right place.

Maltese Cross. The Maltese cross [4], which plays an important part in angular indexing, includes two parts: grooved pulley and crank, seen in Fig. 10. The grooved pulley rotates 60 degrees while the crank rotates 360 degrees. Applying the Maltese cross in angular indexing can reduce the complexity of control system and avoid the cumulative dividing error caused by phase error of stepping motor if used. 


\section{GPS Camera-simulation System}

As mentioned earlier, EV requires GPS technology to obtain location information of BEC stake spots and EV itself. Unfortunately, the introduced demo system is demanded to work in a limited volume $(3 \mathrm{~m} \times 3 \mathrm{~m})$ in which GPS technology is not applicable. Therefore, double-camera technique is employed to simulate GPS technology and achieve positioning and navigation segments of the intelligent EV model.

Image Acquisition and Processing. The simulation of positioning and navigation is implemented through cameras installed above the simulation area. Due to the limitation of cameras' visual angle and work height, one camera fails to cover the whole working region. Thus, to adopt two symmetrical cameras (Fig. 11) is not bad to invert the failure.

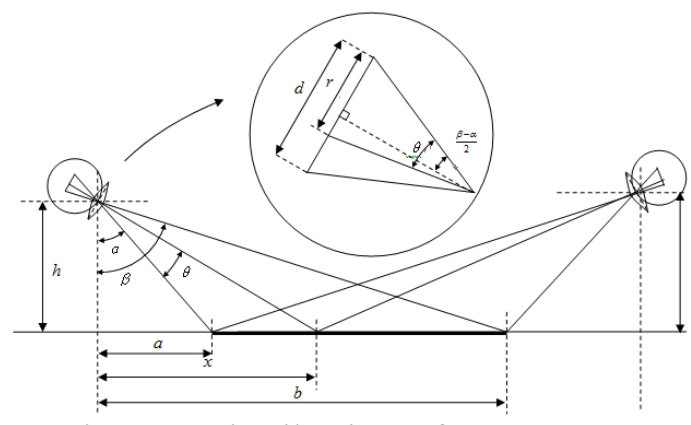

Fig. 11. Distribution of cameras

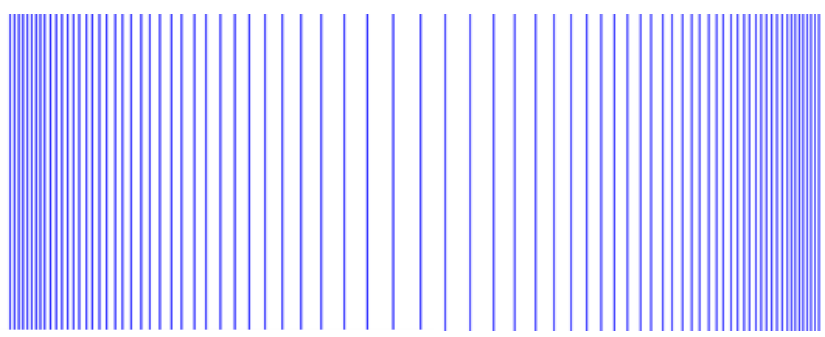

Fig. 12. Revised image

When acquiring images, the cameras collect image spots in a certain resolution and in the way of interlaced scanning. Image sensor chip will convert the gray-level of every image dot to corresponding voltage values that will be exported in the form of video signal. [5]

Image deformation formed by camera collecting should be rectified. Vertical and horizontal deformations are main distortions to be revised in the demo system. Cameras carry out collecting in a $3 \mathrm{~m} \times 3 \mathrm{~m}$ area drawn up many equidistant parallel lines (e.g. 120 lines) and the area will actually take on a thin-center thick-edge appearance and the lines are not parallel in image. Thus, some methods can be used to emend vertical deformation according to some geometric principles. As Fig. 11 shows, $h$ stands for the height of cameras; $b$ represents the distance between apogee and its camera; $x$ symbolizes arbitrary distance from a camera to its apogee; and $\alpha, \beta, \theta$ are corresponding overlooking angle. The geometric relationship of these parameters is as follows:

$$
h=\frac{a}{\tan \alpha}=\frac{b}{\tan \beta}=\frac{x}{\tan (\alpha+\theta)} .
$$

On the sensitive plate of the camera, the length of light-sensitive area is expressed as symbol $d$, which also indicates the overall rows of an image. Making $r$ symbolize one of the rows of the light-sensitive area, on which an image area at $x$ locates, another geometric relationship, Eq. (2), can be obtained.

$$
\frac{\frac{d}{2}}{\tan \left(\frac{\beta-\alpha}{2}\right)}=\frac{r-\frac{d}{2}}{\tan \left(\theta-\frac{\beta-\alpha}{2}\right)} .
$$

The relationship of $r$ and $x$ can be acquired by solving Eq. (1) and Eq. (2), as shown in Eq. (3).

The rectified distribution of rows can be acquired by putting the camera actual parameters $b=300 \mathrm{~cm}, h=150 \mathrm{~cm}$ and $d=275$ into Eq. (3). A result graph of the two cameras rectified by Eq. (3) is plotted by MATLAB and is shown in Fig. 12. Apparently, Harf of Fig. 12 is image of one camera. In a similar way, horizontal deformation could be rectified. 


$$
r=\frac{d \tan \left[\arctan \left(\frac{x \tan \beta}{b}\right)-\frac{\alpha+\beta}{2}\right]}{2 \tan \left(\frac{\beta-\alpha}{2}\right)}+\frac{d}{2}
$$

After doing vertical and horizontal transformation, the collected image will be much closer to the actual image, and a foundation is just made for later simulating the navigation and positioning function.

Simulation of Navigation and Positioning. The navigation and positioning simulation module acts to simulate on-vehicle GPS and displays the results on an upper machine. Positioning function reports location information of BEC stake spots and EV to upper machine and displays them on the display module. Analyzing and comparing position information and battery information obtained by the battery information monitor module, an optimal BEC spot is locked. And then, navigation function works out a route for EV model to arrive the locked BEC spot for battery exchanging. The route can be dynamically displayed [6] on the display module too. A 3D image, shown in Fig. 13, is captured from upper machine and reveals the display effect on the display module.

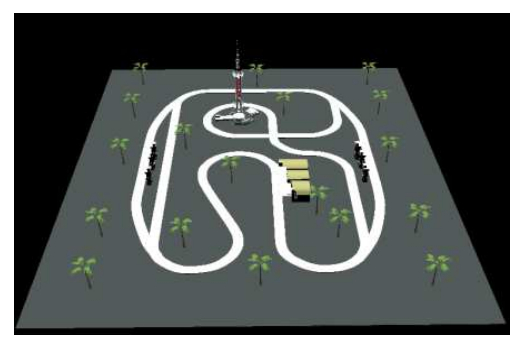

Fig. 13. Display effect of display module

\section{Conclusions}

Under the current circumstances that no interrelated standard for BEC station of EV to abide by, this article followed practical application requirements, considered maturely the developing tendency of future EV and, in a technique level, validated the feasibility of applying self-service BEC stake system to supply EV power by an experimental demo system. The demo system achieved good results in the lab and accomplished system design goals. Looking into the future, with the aggrandizement of EV commercializing scale, to develop synthesized operating systems of BEC stations, which support commercial operations, based on current pattern of BEC stations is necessitated.

\section{References}

[1] X. Han, J. C. Jiang, W.G. Zhang, Simulation of the Operating Plan of E-Bus Charge Station, Microprocessors, 2(2011)88-91.

[2] L.P. Yu, Thinking to construction outlook of electric vehicles charge stations, Auto Industry Research, 7(2010) 21-23.

[3] B.B. Shao, SCM online exploitation methods for embedded applications, Tsinghua University Press, Beijing, 2004.

[4] D.X. Cheng, Handbook of mechanical design, Chemical Industry Press, Beijing, 2007.

[5] S.L. Sun, Z.Q. Ma, W. Tang, Research on gray-level image binarization algorithms, Value Engineering, 5(2010)142-143.

[6] Y.M. Ge, T. Liu, Z.Y. Wang, Video sync separator LM1881 and its application, Applied Science and Technology, 31(2004)20-22. 